\title{
Opções reais sob Incerteza Knightiana na avaliação econômica de projetos de Pesquisa e Desenvolvimento (P\&D)
}

\author{
Luís Alberto Melchíades Leite ${ }^{\mathrm{a*}}$, Leonardo Pereira Santiago ${ }^{\mathrm{b**}}$, José Paulo Teixeira ${ }^{\mathrm{a}}$ \\ a*Pontifícia Universidade Católica do Rio de Janeiro, Rio de Janeiro, RJ, Brasil, lamelchiades@terra.com.br \\ b**Universidade Federal de Minas Gerais, Belo Horizonte, MG, Brasil, lasantiago@ufmg.br
}

\begin{abstract}
Resumo
Este trabalho incorpora tratamento quantitativo da incerteza, no sentido da definição clássica de Frank Knight (1921), a um modelo de avaliação econômica baseado em Opções Reais, analisando suas consequências. 0 conceito knightiano distingue incerteza de risco. Decisões ótimas indicadas pelo Modelo de Opções Reais, uma vez não seguidas pelos decisores, tornam-se escolhas subótimas, sugerindo a presença de elementos de lncerteza Knightiana no ambiente decisório. Decisões subótimas contrariam a regra básica do VPL e, sendo o decisor racional por premissa, existe uma espécie de VPL ex post que se harmoniza à escolha, adequando-se à regra decisória e determinando quantitativamente um grau de aversão à incerteza no decisor. Introduzindo-se no modelo o Valor Esperado de Choquet e um parâmetro representando aversão à incerteza, sob o conceito de probabilidades não aditivas, obtêm-se aproximações de VPLs pós-decisão e graus de aversão à incerteza revelados. A formulação proposta é aplicada em avaliação de projeto de P\&D.
\end{abstract}

Palavras-chave

Decisão sob incerteza. Opções Reais em P\&D. Avaliação econômica de projetos. P\&D incremental. Incerteza Knightiana.

\section{Introdução}

0 economista Frank Knight (1921) estabeleceu uma forte e importante distinção conceitual entre risco e incerteza. De uma maneira simplificada e adequada aos propósitos deste artigo, a distinção de Knight estabelece que risco diz respeito a um estado futuro incerto, definido por meio de variável aleatória cujo espaço probabilístico é perfeitamente conhecido, caso, por exemplo, do lançamento de um dado, ou de uma moeda, ou das loterias de números. Já a incerteza, embora corresponda também a um estado futuro incerto, é definida por meio de variável aleatória, em espaço probabilístico não perfeitamente conhecido, caso, por exemplo, da dúvida sobre a ocorrência de uma terceira guerra mundial no próximo ano, ou a respeito de quando ocorrerá uma nova crise econômica mundial. Savage (1954) fundamentou a modelagem estatística tradicionalmente empregada nos problemas econômicos traduzindo incerteza como um risco sob probabilidades subjetivas, facilitando o seu tratamento quantitativo e "resolvendo" o problema do desconhecimento das probabilidades. Devido a tal simplificação, um tanto atraente sob um ponto de vista prático, o conceito knightiano permaneceu mergulhado em longo ostracismo até o início da década de 1980.

No entanto, o poderoso "atalho" instituído por Savage (1954) no tratamento da incerteza já recebera forte contestação de Ellsberg (1961), que demonstrou que o comportamento dos agentes é diferente quando eles se defrontam com uma situação de incerteza, mostrando que os axiomas por Savage propostos são facilmente violáveis. Ellsberg (1961) também evidenciou que boa parte das pessoas, sob situação de incerteza, não age de acordo com a utilidade esperada (Neumann \& Morgenstern, 1947) e que, se pudessem escolher, evitaria situações de incerteza 
em favor de situações de risco. lsso ficou conhecido como Paradoxo de Ellsberg (Ellsberg, 1961).

Seria fácil justificar tal escolha se fosse possivel comparar as utilidades ou valores esperados nas duas situações, mas não há como fazer inferências de valor em situações de incerteza sem lançar mão das premissas de Savage (1954), em bases bayesianas. No entanto, Schmeidler $(1982,1989)$ conseguiu resolver o paradoxo a partir de uma inovação: o emprego do conceito Capacidades Convexas, que envolve probabilidades subaditivas (também conhecidas como não aditivas), definindo matematicamente uma noção de aversão à incerteza. Uma situação de incerteza pode ser caracterizada por um conjunto de eventos e suas probabilidades sobre um determinando conjunto, não esgotando em 100\% o somatório dessas probabilidades, ou seja, não sendo um espaço aditivo, tal como são as probabilidades no sentido comumente conhecido (i.e., quando somam 100\%).

Seria possível então separar a incerteza do risco, na medida de um certo distanciamento da aditividade da probabilidade em bases convencionais ou bayesianas? Um valor esperado sob incerteza, sendo menor que um valor esperado sob risco, justificaria as atitudes mais prudentes diante da incerteza em agentes que são avessos a ela, como normalmente é o caso em avaliações de projetos de setores industriais tecnologicamente maduros? Isso abriu caminho para o cálculo de valores esperados sob incerteza, resgatando a distinção do conceito knightiano, que prima pela prudência diante do desconhecido. Schmeidler (1982, 1989) foi um pioneiro no emprego da integral de Choquet (1953) envolvendo medidas não aditivas. Nota-se que o desenvolvimento de Choquet (1953) buscou a simplificação de equações diferenciais não lineares em aplicações na física. Schmeidler (1982; 1989) chegou posteriormente a resultado semelhante, trabalhando com teoria da decisão sob incerteza, mas reconhecendo a anterioridade da formulação de Choquet. Em bases subaditivas, com capacidades convexas, portanto, emprega-se o Valor Esperado de Choquet, ou Choquet Expected Utility (CEU) (Gilboa, 2009).

Projetos de Pesquisa e Desenvolvimento (P\&D) são naturalmente dotados de uma carga adicional de risco, de natureza técnica. 0 tratamento metodológico apropriado à avaliação de decisões econômicas sobre essa categoria de projetos requer, como boa prática, a técnica das Opções Reais, como proposto por Huchzermeier \& Loch (2001) e analisado por Santiago \& Vakili (2005). Modelar de forma específica as incertezas técnica e de mercado combinadas, com a disponibilidade de escolhas ao longo do projeto, torna possível a revelação da existência de uma aversão à incerteza no sentido proposto por Knight. Isso se verifica quando o decisor (seja um indivíduo ou um colegiado) não cumpre a decisão ótima recomendada, fazendo uma escolha (exercício de opção) inferior ou subótima, mais cautelosa.

Haveria então uma espécie de valor esperado revelado que, a posteriori, fosse aderente ao resultado final do processo decisório. Por exemplo, seja a seguinte a regra decisória em processo de avaliação de projeto em situação de risco: Iniciar a execução do projeto caso seu VPL esperado seja maior que 0. Pois bem, seja também uma situação em que, após muitos debates numa reunião de colegiado, um projeto de VPL esperado > 0 (sem falhas em sua avaliação) tenha sido adiado ou cancelado por esse colegiado. Admitindo, então, certa primazia à regra decisória, podemos imaginar uma espécie de "VPL esperado pós-decisão", ou "VPL esperado ex post" para esse projeto, que tenha sinal negativo, sendo assim aderente, ou harmonizado, à regra decisória. Claramente, há elementos de incerteza na avaliação desse projeto que não foram devidamente capturados pela modelagem usual de risco. Sob condições de incerteza (considerando-a no sentido de Knight), esse valor esperado, ou algo próximo a ele, é possível de ser calculado por meio do emprego do Valor Esperado de Choquet combinado com um parâmetro adequado da aversão à incerteza.

Um valor esperado normal, sob probabilidades aditivas, é dado pela fórmula:

$$
E[X]=\sum_{i=1}^{n} x_{i} p_{i}
$$

onde $x_{i}(i=1, \ldots, n)$ são os valores da variável aleatória e $\mathrm{p}_{\mathrm{i}}$, suas probabilidades aditivas, ou seja, $\sum_{i=1}^{n} p_{i}<100 \%$. Um tratamento básico de risco em avaliações econômicas se dá quando $x_{i}$ representa um VPL no cenário $i$, com probabilidade de ocorrência $\mathrm{p}_{\mathrm{i}}$, resultando $\mathrm{E}[\mathrm{X}]$ em VPL esperado.

0 Valor Esperado de Choquet pressupõe um cálculo com $\sum_{i=1}^{n} p_{i}<100 \%$ !

Modelos usuais de negócio que tratam dos riscos, incluindo os de Opções Reais, como em Trigeorgis (1996) e Dixit \& Pindyck (1994), empregam distribuições de probabilidades aditivas. 0 universo das finanças corporativas sob incerteza, de maneira geral, está acostumado a lidar com essa forma de definir eventos futuros para o cálculo de valores esperados, na forma da Equação 1. Seria motivadora, então, a possibilidade de aplicar a um problema de avaliação o tratamento da incerteza no sentido de Knight, partindo de algo tão familiar quanto um espaço aditivo de probabilidades já definido para se 
chegar, de forma simplificada, ao cálculo do Valor Esperado de Choquet.

Não há, na proposta dessa visão alternativa, a ideia de estabelecer conflito ou reformular o que já se encontra bem estabelecido na teoria clássica de finanças corporativas, como encontramos em Brealey \& Meyers (2003), por exemplo.

Assim, os fundamentos teóricos amplamente consolidados na orçamentação de capital, onde a avaliação econômica de projetos desempenha papel fundamental, são herdados integralmente [para uma boa visão didática, ver Samanez (2007)]. Em nada se pretende modificar, por exemplo, a consideração usual de risco dada pelo cálculo da devida taxa para o desconto ou capitalização de valores no fluxo de caixa do projeto ao longo do tempo. Assim, o emprego de técnicas consagradas, como o custo médio ponderado do capital - CMPC (em inglês, WACC - Weighted Average Cost of Capital), combinada com o CAPM (Capital Asset Pricing Model), em que se reconhece um prêmio por exposição ao risco sistemático, é preservado. Da mesma forma, não há nenhum questionamento sobre o cálculo do indicador VPL em sua versão determinística. Está no foco da proposta o VPL em sua forma estocástica, onde se consideram múltiplos cenários com probabilidades de ocorrência associadas, empregando-se a fórmula de cálculo de valor esperado, tal como na Equação 1.

Um espaço não aditivo de probabilidades onde $\sum_{i=1}^{n} p_{i}<100 \%$, como será visto mais adiante, não é algo muito confortável de ser definido, principalmente quando é grande a quantidade dos eventos em análise. Basicamente, a diferença na axiomatização em relação ao caso aditivo, ao qual estamos acostumados, está na Convexidade, propriedade que caracteriza a aversão à incerteza. Será visto que existe uma forma de conciliar os espaços probabilísticos aditivo e não aditivo numa modelagem, através de capacidades especiais conhecidas como Capacidades Simples ou Contrações Uniformes. Por meio delas pode-se construir uma espécie de "ponte" entre os mundos de "puro risco" e de "pura incerteza", no sentido de Knight, aproveitando modelos desenvolvidos na base convencional (com probabilidades aditivas).

Adotar a aversão à incerteza no sentido de Knight significa considerar um aspecto da valoração que está mais ligado ao momento tácito da decisão do agente e não ao esforço de calcular propriamente um novo VPL para um investimento que viesse, por exemplo, a ser considerado ex ante. Não temos ainda elementos teóricos suficientes, por enquanto, para considerá-lo com essa abrangência. A proposta visa a análise da atitude revelada do decisor, seja ele um indivíduo ou um colegiado, que considera em sua decisão resultados oferecidos pela teoria de finanças clássica em avaliações de risco, consolidadas e bem estabelecidas no universo corporativo.

Neste artigo vamos caracterizar, de forma simplificada e sem rigor formal, a axiomatização matemática do cálculo de valor esperado sob incerteza no sentido de Knight, que envolve, basicamente, as definições de: Capacidades Convexas, ou probabilidades não aditivas que exibem aversão à incerteza; Capacidades Simples; e Valor Esperado de Choquet. Em seguida, vamos introduzir o Valor Esperado de Choquet em modelo de avaliação econômica de Projetos de P\&D baseado em Opções Reais, especificamente adaptando o cálculo para o VPL com flexibilidade (que é também um VPL esperado) e derivando algumas considerações analíticas dessa implementação. Na sequência vamos aplicar o modelo modificado a um projeto de P\&D e analisar os resultados finais considerando a presença de aversão à incerteza no agente decisor. Finalmente, vamos concluir com a sugestão de uma escala qualitativa para o parâmetro de aversão à incerteza c, componente da nova formulação, e propostas de desdobramentos para futuras pesquisas.

\section{Capacidades Convexas, Valor Esperado de Choquet e grau de aversão à incerteza}

Os conceitos apresentados a seguir são de natureza estritamente estatística e buscam fundamentar a validade do emprego de uma nova fórmula de cálculo para valores esperados: o Valor Esperado de Choquet. Definições mais formalizadas e detalhadas são encontradas em Coimbra-Lisboa (2008). Ao leitor menos interessado nessas demonstrações, recomendamos apenas observar o resultado da Equação 4, na seção 2.3, com a explicação do parágrafo subsequente, e em seguida pular para a seção 3.

0 arcabouço utilizado para explicar a Incerteza Knightiana foi desenvolvido por David Schmeidler (1982; 1989) e por um de seus alunos-colaboradores, Gilboa (Gilboa \& Schmeidler, 1989). Eles utilizaram a integral de Choquet associada a Capacidades Convexas (probabilidades não aditivas que exibem aversão à incerteza). 0 tratamento matemático completo de capacidades é encontrado em Choquet (1953), Dellacherie (1972), Dempster (1967) e Shafer (1976).

\subsection{Capacidades Convexas}

Sejam $\Omega$ um espaço de estados finito e $\varphi$ uma álgebra de eventos mensuráveis de $\Omega$. Uma função conjunto com valores reais v: $\varphi \rightarrow[0,1]$, é considerada 
uma Capacidade Convexa se apresentar as seguintes propriedades:

i. $\mathrm{v}(\varnothing)=0$;

ii. Normalização: $v(\Omega)=1$;

iii.Monotonicidade: $\forall \mathrm{A}, \mathrm{B} \in \varphi, \mathrm{A} \subseteq \mathrm{B} \Rightarrow \mathrm{v}(\mathrm{A}) \leq \mathrm{v}(\mathrm{B})$;

iv. Convexidade: $\forall \mathrm{A}, \mathrm{B} \in \varphi, \mathrm{v}(\mathrm{A} \cup \mathrm{B})+\mathrm{v}(\mathrm{A} \cap \mathrm{B})$ $\leq \mathrm{v}(\mathrm{A})+\mathrm{v}(\mathrm{B})$.

Faz-se importante ressaltar o significado da propriedade iv: trata-se de uma premissa eminentemente econômica, que evidencia a aversão dos agentes econômicos à incerteza. Lembramos que, para o caso aditivo, cumpre-se a igualdade. A letra V é adotada para diferenciar o caso não aditivo de probabilidade (Capacidade Convexa) do caso aditivo, que será referenciado pelo convencional $\mathrm{P}$.

\subsection{Valor Esperado de Choquet}

Seja X uma variável aleatória. X: $\Omega \rightarrow R$, para todos os conjuntos abertos 0 de $R, \mathrm{x}^{-1}(0) \in \varphi$. A integral de Choquet é definida por:

$$
E^{C} X=\int_{-\infty}^{0}(v(x \geq \alpha)-1) d \alpha+\int_{0}^{+\infty} v(x \geq \alpha) d \alpha
$$

O Valor Esperado de Choquet é uma forma de reescrever a integral de Choquet empregando-se uma função real de valor finito (funcional simples).

Sejam: $\Omega=\left\{w_{1}, \ldots, w_{n}\right), \sigma(\Omega)$ uma álgebra de eventos de probabilidades não aditivas, e X uma função de valor real simples.

$\mathrm{X}: \Omega \rightarrow R \mathrm{X}\left(\mathrm{w}_{1}\right)=\mathrm{X}_{1} ; \mathrm{X}\left(\mathrm{w}_{2}\right)=\mathrm{X}_{2} ; \ldots ; \mathrm{X}\left(\mathrm{w}_{\mathrm{n}}\right)=\mathrm{X}_{\mathrm{n}}$;

$V_{k}, \ldots, V_{n}=V\left\{w_{k}, \ldots, w_{n}\right\}$ (Probabilidades não aditivas dos eventos - seguem as propriedades de i a iv);

$\mathrm{X}_{1} \leq \mathrm{X}_{2} \leq \ldots<\mathrm{X}_{\mathrm{n}}$ (ordenação crescente de eventos);

$\mathrm{E}^{\mathrm{c}}[\mathrm{X}]=\mathrm{X}_{1}+\left(\mathrm{X}_{2}-\mathrm{X}_{1}\right) \mathrm{V}_{2} \ldots \mathrm{V}_{\mathrm{n}}+\left(\mathrm{X}_{3}-\mathrm{X}_{2}\right) \mathrm{V}_{3} \ldots$

$V_{n}+\ldots+\left(X_{n}-X_{n-1}\right) V_{n}$

Obs.: $\mathrm{V}_{2} \ldots \mathrm{V}_{\mathrm{n}}$ é a probabilidade conjunta dos eventos $w_{2}$ até $w_{n}, V_{3} \ldots V_{n}$ é a probabilidade conjunta dos eventos $w_{3}$ até $w_{n}$, e assim por diante.

Como exemplo, seja o espaço de probabilidades não aditivas $V$ com três estados da natureza $\left(X_{1}, X_{2}\right.$ e $X_{3}$ ) apresentado na Tabela 1 , baseada em Simonsen \& Werlang (1991), com $X_{1}=1, X_{2}=5, X_{3}=3$ :

Procedendo à reorganização crescente dos eventos, ficamos com: $X^{\prime}{ }_{1}=1, X^{\prime}{ }_{2}=3, X^{\prime}{ }_{3}=5$, o que nos leva a rever as probabilidades, uma vez que os valores mudaram de posição (pois $\mathrm{V}_{12}=\mathrm{V}^{\prime}{ }_{13} ; \mathrm{V}_{13}=\mathrm{V}^{\prime}{ }_{12} \mathrm{e}$ $\mathrm{V}_{23}=\mathrm{V}^{\prime}{ }_{23}$ ), assim, seja a Tabela 2 :

Temos então, aplicando-se a Equação 3:

$\mathrm{E}^{\mathrm{c}}[\mathrm{X}]=1+(3-1)(0.6)+(5-3)(0.2)=2.6$
Tabela 1. Probabilidades não aditivas dos eventos $X_{1}, X_{2}$ e $X_{3}$.

\begin{tabular}{cccc}
\hline & $\mathrm{V}_{1}$ & $\mathrm{~V}_{2}$ & $\mathrm{~V}_{3}$ \\
\hline $\mathrm{V}_{1}$ & $\mathrm{~V}_{11}=0.2$ & $\mathrm{~V}_{12}=0.5$ & $\mathrm{~V}_{13}=0.6$ \\
$\mathrm{~V}_{2}$ & $\mathrm{~V}_{21}=0.5$ & $\mathrm{~V}_{22}=0.2$ & $\mathrm{~V}_{23}=0.6$ \\
$\mathrm{~V}_{3}$ & $\mathrm{~V}_{31}=0.6$ & $\mathrm{~V}_{32}=0.6$ & $\mathrm{~V}_{33}=0.2$ \\
\hline $\mathrm{V}_{123}=1 \mathrm{~V}_{11}+\mathrm{V}_{22}+\mathrm{V}_{33}<1$ & &
\end{tabular}

Tabela 2. Probabilidades não aditivas dos eventos $X^{\prime}, X^{\prime}{ }_{2}$ e $X^{\prime}$.

\begin{tabular}{cccc}
\hline & $\mathrm{V}_{1}$ & $\mathrm{~V}_{2}^{\prime}$ & $\mathrm{V}_{3}^{\prime}$ \\
\hline $\mathrm{V}_{1}^{\prime}$ & $\mathrm{V}_{11}^{\prime}=0.2$ & $\mathrm{~V}_{12}^{\prime}=0.6$ & $\mathrm{~V}_{13}^{\prime}=0.5$ \\
$\mathrm{~V}_{2}^{\prime}$ & $\mathrm{V}_{21}^{\prime \prime}=0.6$ & $\mathrm{~V}_{22}^{\prime 2}=0.2$ & $\mathrm{~V}_{23}^{\prime}=0.6$ \\
$\mathrm{~V}_{3}^{\prime}$ & $\mathrm{V}_{31}^{\prime}=0.5$ & $\mathrm{~V}_{32}^{\prime}=0.6$ & $\mathrm{~V}_{33}=0.2$ \\
\hline $\mathrm{V}^{\prime}{ }_{123}=1 \mathrm{~V}_{11}^{\prime}+\mathrm{V}_{22}^{\prime}+\mathrm{V}_{33}^{\prime}<1$ & &
\end{tabular}

Os autores demonstram um cálculo empregando uma medida equivalente para as verdadeiras probabilidades dos estados, "convertendo" as probabilidades não aditivas em aditivas por meio de um critério de máxima prudência (ponderando os melhores resultados pelas menores probabilidades, progressivamente), chegando ao mesmo resultado. Uma das interpretações das probabilidades não aditivas é que elas representam uma aproximação, ou uma informação incompleta, sobre as verdadeiras e aditivas probabilidades dos estados. Outro exemplo de aplicação é encontrado em Dow \& Werlang (1992).

\subsection{Capacidades simples}

Contudo, a modelagem costumeiramente empregada em gestão de riscos no mundo corporativo não diferencia a incerteza do risco, sendo o universo probabilístico empregado totalmente aditivo. Há uma categoria especial de capacidade que parte de uma medida aditiva e, por meio de uma espécie de "aperto" ou contração dessa medida aditiva, converte um valor esperado "em risco" para um valor esperado "em incerteza", no sentido proposto por Knight. Segue a definição:

Seja $(\Omega, \varphi$, P) um espaço de probabilidades aditivas, $\Omega$ finito e c $\in[0,1]$, e A um evento tal que:

$V(A)= \begin{cases}1, & \text { se } A=\Omega \\ (1-c) P(A) & \text { se } A \neq \Omega\end{cases}$

As capacidades simples, ou contrações uniformes, atendem a todas as propriedades das capacidades convexas (de i a iv). 0 valor esperado de Choquet das capacidades simples ( $\left.E^{c}[X]\right)$ é dado por:

Sejam X: $\Omega \rightarrow \mathfrak{R} \quad \operatorname{MinX}=\min \mathrm{X}(\mathrm{w}) \mathrm{w} \in \Omega$

$$
\operatorname{MaxX}=\max X(w) w \in \Omega
$$

Então: $E^{c}[X]=c \operatorname{Min} X+(1-c) E[X]$

$\mathrm{Ou}-\mathrm{E}^{\mathrm{c}}[-\mathrm{X}]=\mathrm{cMaxX}+(1-\mathrm{c}) \mathrm{E}[\mathrm{X}]$, sendo $\mathrm{E}[\mathrm{X}]$ dado pela Equação 1. 
Ou seja, a integral de Choquet associada a uma contração uniforme pode ser obtida através de uma forma simples, como se fosse uma combinação convexa entre o pior que pode ocorrer (MinX) e o valor esperado original antes de contrair a medida de probabilidade aditiva $(\mathrm{E}[\mathrm{X}])$. Essa foi a ideia que Ellsberg (1961) teve para explicar a maneira como as pessoas escolhem quando se defrontam com uma situação de incerteza, refletindo um comportamento cauteloso, considerando de forma diferenciada o pior que lhes pode ocorrer. Reflete um cálculo de valor esperado coerente com ampla gama de posturas do agente diante da incerteza, envolvendo desde a completa aversão, quando $\mathrm{c}=1$, até a completa indiferença, ou neutralidade, quando $c=0$, lembrando que $c \in[0,1]$ e representa um grau de aversão à incerteza (que será explicado mais adiante na seção 2.4).

Gilboa (2009), argumenta que o Valor Esperado de Choquet, por si só, não pressupõe qualquer noção de aversão à incerteza. Ele não é aplicável quando a medida de probabilidade não seja convexa, ou quando seu núcleo seja vazio. A noção de núcleo de uma capacidade está diretamente ligada à medida de probabilidade aditiva embutida na capacidade. De acordo com sua definição, dada uma capacidade $v$, seu núcleo é o conjunto convexo constituído por todas as medidas de probabilidade finitamente aditivas tais que superem $v$ pontualmente, ou seja:

$N(v)=\{\forall A \in \phi, A \neq \Omega,: P(A) \geq v(A)\}$

onde $\varphi$ representa todas as medidas de probabilidade aditiva.

Portanto, o núcleo de uma capacidade simples é dado por:

$N(v)=\left\{\forall A \in \phi, A \neq \Omega: P^{\prime}(\{A\}) \geq(1-c) P(\{A\})\right\}$

Quando $\mathrm{c}=\mathrm{o}, \mathrm{N}(\mathrm{v})=\{\mathrm{P}(\mathrm{.})\}$, e quando $\mathrm{c}=1$, $N(v)=\varphi$. Segue-se que, sendo $v$ uma capacidade convexa: $v(A)=\operatorname{Min}_{p \in \text { Núcleo(v) }} P(A)$.

Shapley (1972) demonstrou que se uma capacidade é convexa, seu núcleo é convexo e não vazio. Temos, então, que as capacidades simples possuem núcleo não vazio, fundamentando a aplicabilidade do Valor Esperado de Choquet às contrações uniformes. Gilboa \& Schmeidler (1989) mostram que um modelo bastante próximo do comportamento sob incerteza é aquele onde o agente escolhe de modo a maximizar o valor esperado associado ao menor valor, dentre os elementos do núcleo.

\subsection{Grau de aversão à incerteza}

0 parâmetro c, na fórmula do Valor Esperado de Choquet (Equação 4), pressupõe uma medida constante de aversão à incerteza por parte do decisor. Dow
\& Werlang (1992) definem essa medida constante como uma medida de distanciamento da aditividade do evento A e o seu complemento quando há dois estados da natureza como, por exemplo, as situações de "sucesso" e "insucesso". Segue a definição:

Seja $\mathrm{P}$ uma probabilidade (aditiva ou não aditiva) e $\mathrm{A} \subset \Omega$, um evento. $A^{c}$ é o complementar de $A$. A aversão à incerteza de $\mathrm{P}$ em $\mathrm{A}$ é definida por:

$c(P, A)=1-P(A)-P\left(A^{c}\right)$

De acordo com os autores, essa medida reflete um montante de probabilidade perdida pela presença de aversão à incerteza. Reflete o desvio de $\mathrm{P}$ à aditividade de A. Resulta dessa proposição o seguinte lema: c(P, A) $=0$ para todos os eventos $A \subset \Omega$ se, e somente se, $P$ é aditiva.

É natural que $c(P, A)=c\left(P, A^{c}\right)=c$. Ou seja, a medida equivale ao parâmetro $\mathrm{c}$ de aversão à incerteza das capacidades simples, ou contrações uniformes, representando uma postura do agente decisor diante de situações de incerteza. É extensiva também a distribuições com $n$ elementos: Seja $\Omega$ finito com $n$ elementos e seja um espaço de eventos definido pelo conjunto potência de $\Omega, 2^{\Omega}$. Para todo $\omega \in \Omega, P(\{\omega\})=(1-c) / n$, onde $c \in[0,1]$. Para $A \subset \Omega$, $\mathrm{A} \neq \Omega$, definindo $\mathrm{P}(\mathrm{A})=\Sigma_{\omega \in \mathrm{A}} \mathrm{P}(\{\omega\})$, é verificável que $\mathrm{c}(\mathrm{P}, \mathrm{A})=\mathrm{c}, \forall \mathrm{A} \neq \Omega, \phi$.

Exemplo: aproveitando o exercício já feito com o exemplo de Simonsen \& Werlang (1991), $\operatorname{com} X_{1}=1$, $X_{2}=5, X_{3}=3$, digamos esses que sejam eventos de um espaço aditivo com as mesmas probabilidades (i.e., $P_{1}=P_{2}=P_{3}=0.333$ ). Teríamos então $E[x]=3$, vamos assumir também que, sob condições de incerteza, seu valor esperado seja $E^{c}[X]=2.6$, tal como calculado anteriormente no exercício (Equação 3a). Assumindo esses valores, podemos aplicar o Valor Esperado de Choquet para contrações uniformes (Equação 4) para calcular um grau de aversão à incerteza c para esse agente:

$\mathrm{E}^{\mathrm{c}}[\mathrm{X}]=\mathrm{cMinX}+(1-\mathrm{c}) \mathrm{E}[\mathrm{X}]=>$

$2,6=\mathrm{cx} 1+(1-\mathrm{c}) \times 3=>\mathrm{c}=0.2$

\section{Um modelo de opções reais para P\&D com aversão à incerteza}

Santiago \& Bifano (2005) propõem uma aplicação prática de modelo de avaliação econômica de projetos de P\&D baseados na proposição seminal de Huchzermeier \& Loch (2001). 0 modelo trata uma das grandes fontes de incerteza (no sentido de risco subjetivo) em projetos de $P \& D$, que é o desempenho técnico do desenvolvimento da nova tecnologia. É adotada uma equação de programação dinâmica 
para a valoração de nós intermediários (dinâmica de transição - Equação 6) de uma árvore multinominal recombinante que define um processo endógeno de difusão de uma variável aleatória, em casos de "sucesso" e "insucesso" em estágios, representando um desempenho técnico $X_{t}$, detalhado a seguir:

$$
\left.V_{t}\left(X_{t}\right)=\max _{u_{t}}\left\{\left(-C_{t}\left(u_{t}\right)+\frac{1}{1+r} E\left[\theta_{t+1}\right)\right]\right), 0\right\}
$$

onde: $\mathrm{t}=$ tempo correspondente ao estágio, variando de 0 a $\mathrm{T}$ (tempo de lançamento da tecnologia); $V_{t}=$ VPL com flexibilidade (neutro ao risco) no estágio $t ; E\left[\theta_{t+1}\right]=p\left(V_{t+1}\left(X_{t+1}^{\prime}\right)\right)+(1-p)\left(V_{t+1}\left(X^{\prime \prime}{ }_{t+1}\right)\right)=$ valor esperado neutro ao risco com base no desempenho técnico no estágio $t+1$ e na decisão tomada em $t$, ou seja: $\theta_{t+1}=f\left(X_{t+1}, u_{t}\right) ; X_{t+1}$ será detalhado mais adiante;

$p$ é a probabilidade de sucesso; e (1-p), a de insucesso; $u_{t}=$ decisão ou opção a seguir no estágio t (Melhorar/Continuar/Abandonar ou Parar); $\mathrm{C}_{t}\left(\mathrm{u}_{\mathrm{t}}\right)=$ custo da decisão, de acordo com o que segue:

$C_{t}\left(u_{t}\right)=\left\{\begin{array}{cl}0, & \text { se } u_{t}=\text { Abandonar } \\ c(t), & \text { se } u_{t}=\text { Continuar } \\ c(t)+\alpha(t), & \text { se } u_{t}=\text { Melhorar }\end{array}\right.$

$r=$ taxa de juros livre de risco (o risco técnico não é correlacionado aos movimentos de mercado, portanto, passível de ser minimizado por diversificação).

Em cada um destes estágios, o tomador de decisão tem a flexibilidade de exercer otimamente as opções sobre o projeto. No estágio seguinte, $t+1$, o estado de desempenho do projeto depende do estado de desempenho no estágio $t\left(X_{t}\right)$, da decisão gerencial $\left(u_{t}\right)$, de um deslocamento $\mathrm{K}\left(\mathrm{u}_{\mathrm{t}}\right)$, onde $\mathrm{K}$ (continuar) $=0 \mathrm{e}$ $\mathrm{K}($ melhorar $)=1$, e da incerteza representada por $\omega_{t}$, uma variável aleatória com média 0 e variância constante.

Dessa maneira, $X_{t+1}$ pode ser representado pela seguinte equação:

$X_{t+1}=\left\{\begin{array}{cccc}X_{t}+k\left(u_{t}\right)+\omega_{t} & u_{t}= & \text { Continuar }, \text { Melhorar } \\ \text { Parar } & u_{t}= & \text { Parar }\end{array}\right.$

Os nós terminais da árvore são definidos por valores de mercado para a tecnologia implantada (curva de payoff), que devem considerar precificação neutra ao risco.

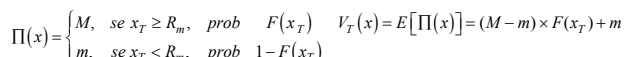

onde: M e m são valores máximos e mínimos pagos pelo mercado para os diversos desempenhos técnicos finais possíveis no desenvolvimento; $\mathrm{Rm}$ é um requisito médio de mercado para a tecnologia; $F\left(x_{T}\right)$ e 1 - $F\left(x_{T}\right)$ são probabilidades aditivas para os dois estados da natureza possíveis ao desempenho técnico final do projeto (igual ou acima e abaixo dos requisitos de mercado, respectivamente).

Um esquema geral simplificado do processo é representado na Figura 1, que mostra um desenvolvimento em árvore binomial (não necessariamente as árvores devem ser binomiais), com as decisões ótimas $u_{t}$ em cada nó.

Os nós intermediários são calculados por backward induction, dos nós finais até o nó inicial, $\mathrm{V}_{0}\left(\mathrm{X}_{0}\right)$, no momento $t_{0}$. Os valores esperados dos nós terminais [os diversos $\mathrm{V}_{\mathrm{T}}\left(\mathrm{X}_{\mathrm{T}}\right)$ ] são descontados em um período pela taxa de juros livre de risco $r$, para logo em seguida ser abatido o custo da decisão [vide Equação 6] do estágio imediatamente anterior, para obter-se os valores dos nós do penúltimo estágio. Esse procedimento é feito para as decisões Melhorar e Continuar, para todos os nós dos estágios anteriores, sendo que o máximo entre esses valores de decisão e 0 será o resultado final de $V_{t}$ do nó calculado. Para um resultado 0 , a decisão ótima será Parar, representada pela letra P. Para o caso de o máximo ser o valor da opção Continuar, a representação será pela letra $\mathrm{C}$, sendo a mesma consideração extensiva à opção Melhorar, na letra M. 0 procedimento é repetido até o nó inicial.

Ao longo da expansão da árvore são considerados dois estados futuros possíveis: Sucesso, com probabilidade $p$, e insucesso, com probabilidade 1-p, em espaço de probabilidades aditivas. Esse modelo representa uma forma adequada de lidar com o risco endógeno na presença de flexibilidade gerencial, seu resultado final é um valor presente líquido com flexibilidade (VPL FLEX) e neutro ao risco. No momento $\mathrm{t}_{0}$, a decisão diz respeito ao início do projeto, que acontece nos casos de $u_{t}=$ Continuar ou Melhorar.

A aplicação do conceito knightiano de incerteza, separando a incerteza do risco, possibilita considerar a presença de um grau constante de aversão à incerteza no ambiente da pesquisa tecnológica, numa dimensão tácita do ato decisório. É factível

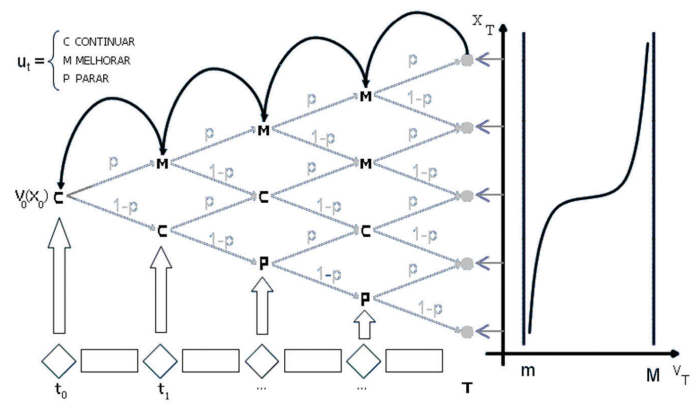

Figura 1. Esquema geral de Modelo de Valoração Econômica de Projetos de P\&D. Fonte: adaptado de Petrobras \& Universidade Federal de Minas Gerais (2008). 
por meio da transformação da variável aleatória $X_{t}$, que representa tecnicamente o desenvolvimento tecnológico, numa capacidade simples, ou contração uniforme, conforme definido na seção 2.3 , com o cálculo de valor esperado dado pela Equação 4. 0 resultado para a formulação da valoração dos nós interiores do modelo, anteriores aos nós terminais, nos dá o VPL FLEX Choquet, assim definido:

$$
\begin{aligned}
V_{t}^{C}\left(X_{t}\right)=\operatorname{Max}_{u_{t}}\left\{\left(-C_{t}\left(u_{t}\right)+\frac{1}{(1+r)}\left(c \operatorname{Min} \theta_{t+1}+(1-c) E\left[\theta_{t+1}\right]\right)\right), 0\right\} \\
\mathrm{c} \epsilon[0,1]
\end{aligned}
$$

Lembrando que $E\left[\theta_{t+1}\right]$ e Min $\theta_{t+1}$ também dependem de $u_{t}$. Pode-se considerar que o Valor Esperado de Choquet presente na nova formulação é dado por: $\left.E^{c}\left[\theta_{t+1}\right]=c \operatorname{Min} \theta_{t+1}+(1-c) E\left[\theta_{t+1}\right]\right)$. Segue-se, naturalmente, que quando $c=0, V_{t}^{c}\left(X_{t}\right)=V_{t}\left(X_{t}\right)$, e quando $\mathrm{c}=1, V_{t}^{C}\left(X_{t}\right)=\operatorname{Max}_{u_{t}}\left\{\left(-C_{t}\left(u_{t}\right)+\frac{1}{(1+r)}\left(\operatorname{cMin}_{t+1}\right)\right), 0\right\}$.

A Equação 10 representa o emprego do Valor Esperado de Choquet, dado pela Equação 4, na Equação 6. Na ausência de incerteza $(c=0)$, o valor esperado não muda em relação à formulação original, mas na presença de total aversão à incerteza $(c=1)$, o valor esperado é substituído pelo valor mínimo da distribuição aditiva, refletindo a postura de prudência máxima do agente.

É possível estender, opcionalmente, a aversão à incerteza à avaliação de mercado da tecnologia comercializada, por meio da Equação 11. Entendemos, em princípio, que não seja adequado, por tratar-se a incerteza sobre um projeto de P\&D característica estritamente ligada ao processo de desenvolvimento da tecnologia e não ao seu lançamento no mercado, já pronta.

$$
\begin{gathered}
V_{T}^{C}(x)=E^{C}[\Pi(x)]=c m+(1-c)\left[(M-m) F\left(x_{T}\right)+m\right] \\
\mathrm{c} \in[0,1]
\end{gathered}
$$

Da mesma forma, a Equação 11 representa o emprego do Valor Esperado de Choquet, dado pela Equação 4 na Equação 9. Segue-se também naturalmente que, quando $\mathrm{c}=0, V_{T}^{c}\left(X_{t}\right)=V_{T}\left(X_{T}\right)$, e quando $\mathrm{c}=1, V_{T}^{c}\left(X_{T}\right)=m$.

Assim, o modelo adaptado ao tratamento da incerteza no sentido de Knight adota as Equações 10 e 11 (esta última caso se queira estender a abrangência da incerteza à tecnologia desenvolvida levada ao mercado) em lugar das Equações 6 e 9, com o parâmetro adicional c indicando o grau de aversão à incerteza do agente decisor. 0 parâmetro c vai de 0 , representando ausência de aversão à incerteza, a 1, representando presença de total aversão à incerteza.

\subsection{Implicações analíticas do novo modelo}

A nova formulação introduz, basicamente, o parâmetro de aversão à incerteza c e o destaque para o valor mínimo da distribuição de valores esperados, em bases aditivas, das opções para o estágio seguinte. Algumas conclusões interessantes podem ser tiradas a partir das relações entre as variáveis críticas na determinação do valor presente líquido com flexibilidade de cada opção no momento da decisão em t. A opção ótima será determinada pela opção de maior valor dentre as três disponíveis no modelo (Parar, Continuar ou Melhorar). Examinando a nova formulação, destacamos que essas variáveis críticas são:

- 0 custo da decisão $C_{t}$;

- 0 valor esperado descontado $(1 /(1+r)) . E\left(\theta_{t+1}\right)$;

- 0 valor futuro mínimo descontado $(1 /(1+r)) \cdot \operatorname{Min}\left(\theta_{t+1}\right)$.

É possível o estabelecimento de relações genéricas entre essas variáveis, que podem determinar, a priori, a decisão ótima a ser tomada em t. Dependendo do grau de aversão à incerteza presente no agente, responde-se, por meio dessas relações, a perguntas do tipo: Sob total aversão à incerteza do decisor $(c=1)$, que relação entre o custo da decisão $\left(C_{t}\right)$, o valor esperado descontado - $[1 /(1+r)] \cdot E\left(\theta_{t+1}\right)$ - e o valor mínimo futuro descontado - $[1 /(1+r)] \operatorname{Min}\left(\theta_{t+1}\right)$ - garantiria o início de um projeto? Valor ótimo, nesse caso, determinado por uma opção Continuar ou Melhorar. Ou então: Com algum grau de aversão à incerteza (c $>0$ ), qual a relação entre as variáveis que determinaria a parada ótima do projeto?

Primeiramente, é preciso investigar cada termo da formulação, para cada uma das decisões possíveis, de forma que tenhamos, separadamente, os custos, os valores esperados descontados e os valores mínimos futuros descontados das opções ativas Continuar e Melhorar. A opção Parar é definida por exclusão, ou seja, é ótima e de valor 0 quando os valores das opções ativas são negativos, sendo seu custo predefinido como 0. Segue o detalhamento:

- $\mathrm{V}_{\mathrm{t}}^{\mathrm{c}} \mathrm{C} \equiv \mathrm{VPL}$ com flexibilidade Choquet do projeto em t, na decisão Continuar;

- $\mathrm{V}_{\mathrm{t}}{ }^{\mathrm{C}} \mathrm{M} \equiv \mathrm{VPL}$ com flexibilidade Choquet do projeto em t, na decisão Melhorar;

- $\operatorname{Min} \theta_{t+1} \mathrm{M} \equiv$ Valor mínimo do projeto em $\mathrm{t}+1$, na decisão Melhorar;

- $\operatorname{Min} \theta_{t+1} \mathrm{C} \equiv$ Valor mínimo do projeto em $\mathrm{t}+1$, na decisão Continuar;

- $\mathrm{E}\left[\theta_{\mathrm{t}+1} \mathrm{M}\right] \equiv$ Valor esperado do projeto em $\mathrm{t}+1$, na decisão Melhorar;

- $\mathrm{E}\left[\theta_{\mathrm{t}+1} \mathrm{C}\right] \equiv$ Valor esperado do projeto em $\mathrm{t}+1$, na decisão Continuar; 
- $\mathrm{E}^{\mathrm{C}}\left[\theta_{\mathrm{t}+1} \mathrm{M}\right] \equiv$ Valor esperado Choquet do projeto em $\mathrm{t}+1$, na decisão Melhorar;

- $\mathrm{E}^{\mathrm{C}}\left[\theta_{\mathrm{t}+1} \mathrm{C}\right] \equiv$ Valor esperado Choquet do projeto em $\mathrm{t}+1$, na decisão Continuar;

(lembrando que $\mathrm{E}^{\mathrm{C}}\left[\theta_{\mathrm{t}+1}\right]=\mathrm{cMin} \theta_{\mathrm{t}+1}+(1-\mathrm{c})$

$$
\left.\mathrm{E}\left[\theta_{\mathrm{t}+1}\right], c \in[0,1]\right)
$$

- $\mathrm{C}_{\mathrm{t}} \mathrm{C} \equiv$ Custo, no tempo t, da decisão Continuar;

- $\mathrm{C}_{\mathrm{t}} \mathrm{M} \equiv$ Custo, no tempo t, da decisão Melhorar.

Regra de decisão ótima :

Parar $=>\operatorname{Max}\left(\mathrm{V}_{\mathrm{t}}{ }^{\mathrm{c}} \mathrm{M}, \mathrm{V}_{\mathrm{t}}^{\mathrm{c}} \mathrm{C}, 0\right)=0$

Continuar $=>\operatorname{Max}\left(\mathrm{V}_{\mathrm{t}}^{\mathrm{c}} \mathrm{M}, \mathrm{V}_{\mathrm{t}}^{\mathrm{c}} \mathrm{C}, 0\right)=\mathrm{V}_{\mathrm{t}}{ }^{\mathrm{c}} \mathrm{C}$

Melhorar $=>\operatorname{Max}\left(\mathrm{V}_{\mathrm{t}}^{\mathrm{c}} \mathrm{M}, \mathrm{V}_{\mathrm{t}}{ }^{\mathrm{c}} \mathrm{C}, 0\right)=\mathrm{V}_{\mathrm{t}}^{\mathrm{c}} \mathrm{M}$

A valoração dos nós interiores do modelo é determinada pela comparação dos valores das decisões ativas entre si. Dessa forma, a Equação 10, adaptada às definições feitas acima, ficaria assim:

$V_{t}^{c} C\left(X_{t}\right)=-C_{t} C+\frac{1}{(1+r)} E^{c}\left[\theta_{t+1} C\right]$

para a opção Continuar, e, sendo $\mathrm{E}^{\mathrm{C}}\left[\theta_{\mathrm{t}+1} \mathrm{C}\right]=\mathrm{cMin} \theta_{\mathrm{t}+1} \mathrm{C}+(1-\mathrm{c}) \mathrm{E}\left[\theta_{\mathrm{t}+1} \mathrm{C}\right], \mathrm{c} \in[0,1]$

$V_{t}^{c} M\left(X_{t}\right)=-C_{t} M+\frac{1}{(1+r)} E^{c}\left[\theta_{t+1} M\right]$

para a opção Melhorar, sendo $\mathrm{E}^{\mathrm{C}}\left[\theta_{\mathrm{t}+1} \mathrm{M}\right]=\mathrm{cMin} \theta_{\mathrm{t}+1} \mathrm{M}+(1-\mathrm{c}) \mathrm{E}\left[\theta_{\mathrm{t}+1} \mathrm{M}\right], \mathrm{c} \in[0,1]$

Os resultados das relações relevantes entre as variáveis críticas, para a determinação de cada decisão ótima e considerando o nível de aversão à incerteza, podem ser visualizados no quadro resumo da Tabela 3, que passaremos a explicar. Observando-se, primeiramente, os resultados para a decisão ótima $\left(u_{t}\right)$ Parar (linha 2), na ausência de aversão à Incerteza Knightiana ( $c=0$, ou coluna 2 ), verifica-se que os valores esperados futuros das opções ativas Melhorar e Continuar não superam seus respectivos custos, resultando em valores presentes líquidos negativos para essas escolhas. Consequentemente, o ótimo aponta para a opção Parar, cujo valor presente é 0 . $\mathrm{Na}$ presença de total aversão à incerteza no sentido de Knight ( $c=1$, ou coluna 3), essa relação passa a ser determinada pelos valores mínimos das distribuições futuras de cada decisão. Já com uma aversão à incerteza intermediária, entre 0 e 1 (coluna 4), a relação determinante combina linearmente valores mínimos e os valores esperados, atribuindo um peso c ao primeiro, e (1-c) ao segundo.

Para um ótimo na opção $\left(u_{t}\right)$ Melhorar, na ausência de aversão à incerteza $(c=0)$, a diferença descontada entre os valores esperados das opções Melhorar e Continuar, deve superar a diferença entre seus respectivos custos, resultando num valor presente maior para a primeira. Seguindo a mesma lógica, na presença de total aversão a incerteza (c = 1), a diferença descontada de valor futuro mínimo, com relação ao mesmo valor da opção Continuar, deve superar a diferença entre seus respectivos custos. Já

Tabela 3. Quadro resumo das relações entre as variáveis que determinam as decisões ótimas na presença de aversão à incerteza no sentido de Knight.

\begin{tabular}{|c|c|c|c|}
\hline $\begin{array}{c}u_{t} \\
\text { Decisão } \\
\text { ótima }\end{array}$ & $\begin{array}{c}c=0 \\
\text { Sem aversão à incerteza } \\
\text { (puro RISCO) }\end{array}$ & $\begin{array}{c}\mathrm{c}=1 \\
\text { Com total aversão à incerteza } \\
\text { (pura INCERTEZA) }\end{array}$ & $\begin{array}{c}0<\mathrm{c}<1 \\
\text { Mix entre RISCO e INCERTEZA }\end{array}$ \\
\hline \multirow[t]{2}{*}{ PARAR } & $\begin{array}{l}{\left[\frac{1}{(1+r)}\right] E\left[\theta_{t+1} M\right] \leq C_{t} M} \\
{\left[\frac{1}{(1+r)}\right] E\left[\theta_{t+1} C\right] \leq C_{t} C}\end{array}$ & $\begin{array}{l}{\left[\frac{1}{(1+r)}\right] \operatorname{Min} \theta_{t+1} M \leq C_{t} M} \\
{\left[\frac{1}{(1+r)}\right] \operatorname{Min} \theta_{t+1} C \leq C_{t} C}\end{array}$ & $\begin{array}{l}{\left[\frac{1}{(1+r)}\right]\left(c \operatorname{Min} \theta_{t+1} M+(1-c) E\left[\theta_{t+1} M\right]\right) \leq C_{t} M} \\
e \\
{\left[\frac{1}{(1+r)}\right]\left(c \operatorname{Min} \theta_{t+1} C+(1-c) E\left[\theta_{t+1} C\right]\right) \leq C_{t} C}\end{array}$ \\
\hline & \multicolumn{3}{|c|}{$\operatorname{Max}\left(V_{t}^{C} M, V_{t}^{C} C, 0\right)=0$} \\
\hline \multirow[t]{2}{*}{ MELHORAR } & $\begin{array}{c}{\left[\frac{1}{(1+r)]}\right]\left(E\left[\theta_{t+1} M\right]-E\left[\theta_{t+1} C\right]\right)} \\
> \\
{\left[C_{t} M-C_{t} C\right]}\end{array}$ & $\begin{array}{c}{\left[\frac{1}{(1+r)}\right]\left(\operatorname{Min}_{t+1} M-\operatorname{Min}_{t+1} C\right)} \\
> \\
{\left[C_{t} M-C_{t} C\right]}\end{array}$ & $\begin{array}{c}{\left[\frac{1}{(1+r)}\right]\left(c\left(\operatorname{Min}_{t+1} M-M \operatorname{Min} \theta_{t+1} C\right)+(1-c)\left(E\left[\theta_{t+1} M\right]-E\left[\theta_{t+1} C\right]\right)\right)} \\
> \\
{\left[C_{t} M-C_{t} C\right]}\end{array}$ \\
\hline & \multicolumn{3}{|c|}{$\operatorname{Max}\left(V_{t}^{C} M, V_{t}^{C} C, 0\right)=V_{t}^{C} M$} \\
\hline \multirow[t]{2}{*}{ CONTINUAR } & $\begin{array}{c}{\left[\frac{1}{(1+r)]}\right]\left(E\left[\theta_{t+1} M\right]-E\left[\theta_{t+1} C\right]\right)} \\
\leq \\
{\left[C_{t} M-C_{t} C\right]}\end{array}$ & $\begin{array}{c}{\left[\frac{1}{(1+r)}\right]\left(\operatorname{Min}_{t+1} M-\operatorname{Min} \theta_{t+1} C\right)} \\
\leq \\
{\left[C_{t} M-C_{t} C\right]}\end{array}$ & $\begin{array}{c}{\left[\frac{1}{(1+r)}\right]\left(c\left(\operatorname{Min}_{t+1} M-\operatorname{Min} \theta_{t+1} C\right)+(1-c)\left(E\left[\theta_{t+1} M\right]-E\left[\theta_{t+1} C\right]\right)\right)} \\
\leq \leq \leq \\
{\left[C_{t} M-C_{t} C\right]}\end{array}$ \\
\hline & \multicolumn{3}{|c|}{$\operatorname{Max}\left(V_{t}^{C} M, V_{t}^{C} C, 0\right)=V_{t}^{C} C$} \\
\hline
\end{tabular}


com uma aversão à incerteza intermediária, entre 0 e 1, a diferença passa a ser sobre uma combinação linear entre valor esperado e valor futuro mínimo de cada opção, que deve, também, superar a diferença entre seus custos.

Para um ótimo $\left(u_{t}\right)$ na opção Continuar basta inverter a desigualdade das comparações anteriores (opção ótima Melhorar), considerando que, em caso de igualdade, a vantagem é da opção Continuar, pois é razoável considerar que não é interessante incorrer num custo maior (Melhorar) para obter o mesmo valor presente líquido de Continuar. Importante observar, também, que as relações entre as variáveis no caso das opções ótimas Melhorar e Continuar são absolutamente determinantes desde que não se cumpram concomitantemente às relações da opção ótima Parar. Por exemplo, sob total aversão à incerteza, acontecendo um ótimo na opção Parar, poderá ser cumprida também a relação da opção ótima Continuar, pois no caso em que $V_{t}^{c} M$ e $V_{t}^{c} C$ são negativos, a diferença entre os mínimos de $\theta_{t+1} \mathrm{M}$ e $\theta_{t+1} \mathrm{C}$ poderá ser inferior à diferença entre os custos das decisões. Ou seja, em acontecendo um ótimo na opção Parar, as demais opções devem ser descartadas, mesmo que suas relações de otimalidade se cumpram. Isso ficará mais claro na aplicação do modelo descrita adiante.

Uma situação de particular interesse é aquela que garante a continuidade de um determinado projeto sob uma condição de máxima severidade (decisão ótima no gate igual a, pelo menos, Continuar, com o máximo de aversão à incerteza, ou seja, com $\mathrm{c}=1$ ). Visto de uma forma mais simples e direta, considerando um ótimo dado pela decisão Continuar, cumpre-se a relação:

$$
C_{t} C \leq \frac{1}{(1+r)} \operatorname{Min}_{t+1} C
$$

Ou seja, o valor mínimo da distribuição de valores futuros em $\mathrm{t}+1$ descontado não é inferior ao custo da decisão em t. Essa relação poderia ser chamada de Espaço de Continuidade dos Projetos. Graficamente, teríamos o que mostra a Figura 2. A área acima da reta de $45^{\circ}$ (incluindo-a) determina o espaço de valores que garantem a continuidade do projeto ao próximo estágio e a área abaixo da reta, o de valores que asseguram a sua parada, ou interrupção ótima.

Poderíamos estar interessados, também, em saber se há alguma configuração de valores esperados e valores mínimos futuros descontados que possa anular a presença de algum grau de aversão à incerteza no agente, para qualquer decisão, ou seja, se essa configuração de valores determinaria a mesma decisão $\left(u_{t}\right)$ para quaisquer valores possíveis de c. Partindo da situação em que a decisão nos dois modelos (com e sem o tratamento de aversão à incerteza) seja a mesma, temos que:

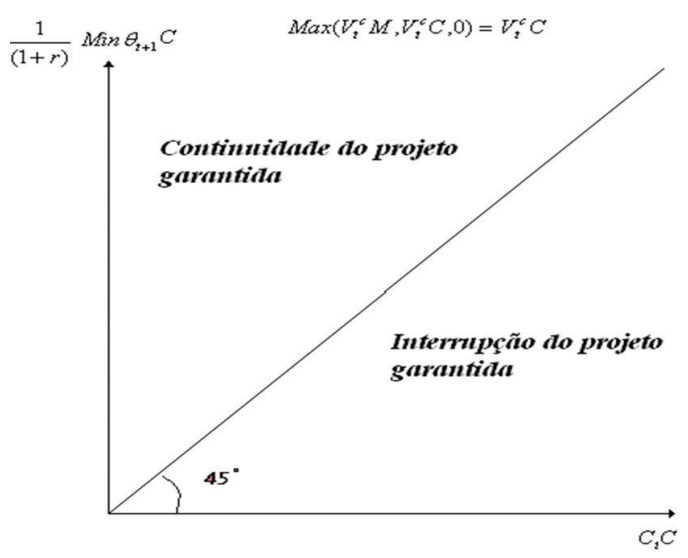

Figura 2. Espaços de continuidade e descontinuidade dos projetos.

$$
u_{t}\left(V_{t}^{c}\left(X_{t}\right)\right)=u_{t}\left(V_{t}\left(X_{t}\right)\right)
$$

Vamos verificar que a igualdade se cumpre para o caso óbvio em que $c=0$ e na situação em que 0 valor mínimo da distribuição futura se iguale ao valor esperado, ou seja, quando:

$$
\operatorname{Min} \theta_{t+1}=E\left[\theta_{t+1}\right]
$$

Em particular, essa configuração de valores torna totalmente sem efeito a presença do parâmetro c. Faz todo o sentido, pois uma vez eliminada a variabilidade do valor futuro, é eliminado o seu risco e não há incerteza quando não há risco, pois incerteza nada mais é, na conceituação que consideramos, do que um risco "não aditivo".

\section{Aplicação e resultados}

Vamos apresentar agora um exemplo de aplicação do modelo aqui sugerido, considerando a valoração de um projeto de $\mathrm{P} \& \mathrm{D}$ que visa ganhos incrementais de desempenho em processo industrial. A descrição completa do projeto assim como de sua avaliação primária com o modelo de opções reais que nos serviu de base encontra-se em leite et al. (2012).

A valoração primária ampla do projeto trata também o risco em seu tempo de desenvolvimento, atribuindo à duração desses tempos distribuições de probabilidade triangulares (para maiores detalhes sobre a incerteza do tempo, ver Silva \& Santiago, 2009). Vamos desconsiderá-la neste artigo, ficando apenas com os resultados apresentados em avaliação parcial que considera uma duração determinística dos tempos de duração de estágio.

0 projeto refere-se à derivação de um novo modelo matemático de fluidodinâmica computacional. Como "cérebro" de um simulador de processo, a nova 
modelagem visa obter um ganho incremental no rendimento da conversão de matéria-prima bruta sólida em produto final líquido, em processo produtivo de indústria extrativista. A expectativa inicial é a obtenção de um ganho de eficiência em torno de 0,1\%, mas podendo chegar a $0,4 \%$ em expectativa mais otimista, ou a $-0,05 \%$, em expectativa mais pessimista. Essa situação é tratada na árvore de incerteza mostrada na Figura 3. Os resultados da avaliação do projeto em Leite et al. (2012) correspondem ao que seria obtido no modelo modificado aqui sugerido (com Incerteza Knightiana), parametrizado em c $=0$, ou seja, sem aversão à incerteza no sentido de Knight. A probabilidade de sucesso p é estabelecida em 50\%.

A árvore multinomial da variável $X_{t}$ que representa a incerteza técnica pode ser vista detalhadamente a seguir. As opções Continuar e Melhorar alcançam um diferente conjunto de nós futuros, sendo a opção Melhorar mais abrangente, em função de, sob maior aporte de recursos (os custos são diferenciados por opção), alcançar-se um desempenho diferenciado (melhorado), de acordo com as premissas do modelo.

Nas Figuras 4a, b são apresentados resumos da avaliação com c $=0$, com a descrição de duas árvores de incerteza, com opções ótimas $\left(u_{t}\right)$, desempenhos técnicos $\left(X_{t}\right)$, Valores Esperados Choquet $\left(V_{t}\right)^{c}$, custos das opções etc. Para o cálculo dos VPLs dos nós terminais $\left(\mathrm{V}_{T}\right)$ foram considerados os ganhos líquidos incrementais obtidos pelos produtos vendidos pela empresa em diferentes quantidades (maiores em desempenhos finais superiores e menores em desempenhos finais inferiores), em função das mudanças de eficiência técnica no processo produtivo.

Os preços foram projetados por meio de projeção de série histórica, considerando uma valoração neutra ao risco, e o fluxo de caixa incremental de cinco anos

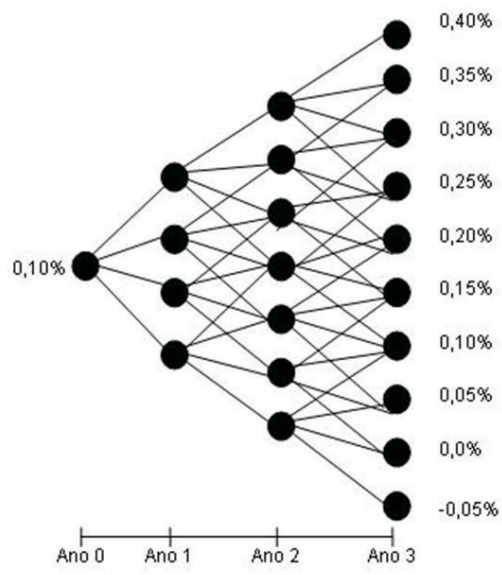

(tempo de depreciação do simulador) foi descontado pela taxa livre de risco, arbitrada em 6\%, e também empregada no cálculo dos VPLs FLEX [intermediários e inicial, $\left.\left(V_{t}{ }^{c}\right)\right]$ do projeto de P\&D.

A primeira árvore (Figura 4a) leva em conta as flexibilidades do projeto, em escolha ótima de opções, e a segunda (Figura 4b) trata-o de forma inflexível, sem o exercício de opções (ou com a opção obrigatória Continuar em todos os estágios), para o cálculo do VPL simples (sem flexibilidade). Há uma ligeira diferença entre os números finais da avaliação de Leite et al. (2012) e os reproduzidos com o novo modelo aqui apresentado, devido a esse último ter sido implementado em planilha eletrônica Excel e o primeiro em aplicativo escrito em $\mathrm{C}++$. São erros de arredondamento acumulados, exemplo: em Leite et al. (2012), o VPL FLEX é de R\$162,07 mil, enquanto que o calculado para este artigo é de R\$162,25 mil.

$\mathrm{Na}$ visão com flexibilidade (Figura 4a), o valor do projeto (VPL flex) é de R\$162,25 mil, com a opção ótima Melhorar. Consequentemente, o valor da opção é de $R \$ 230,28 \mathrm{mil}$ [R\$ 162,25 mil - (-R\$ 68,03 mil)]. $\mathrm{Na}$ visão sem flexibilidade (Figura $4 \mathrm{~b}$ ), o projeto jamais seria iniciado, pois o VPL é negativo em $\mathrm{R} \$ 68,03$ mil. As avaliações foram feitas considerando-se um decisor sem aversão à incerteza no sentido de Knight, o que se materializa com a fixação do parâmetro c em 0.

Considerando-se agora o outro extremo da aversão à incerteza, onde $\mathrm{c}=1$ (total aversão à incerteza no sentido de Knight), os mesmos cálculos de VPL com e sem flexibilidade, nesse modelo de opções reais com Incerteza Knightiana, apresentam os seguintes resultados:

Quais sejam, VPL FLEX $=\mathrm{R} \$ 0,00$ (Figura 5a) e $\mathrm{VPL}=\mathrm{R} \$-522,81$ (Figura 5b). 0 projeto perde sua atratividade econômica na visão com flexibilidade e

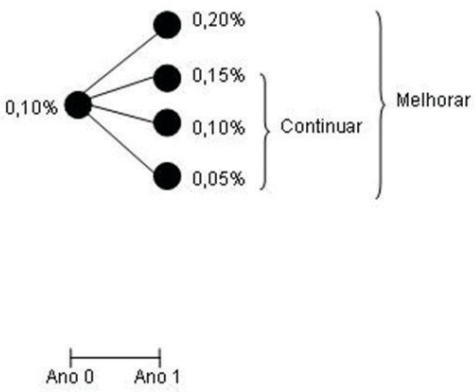

Figura 3. Incerteza técnica detalhada do projeto de fluidodinâmica computacional. Fonte: Leite et al. (2012). 
a opção ótima é Parar ou simplesmente não iniciá-lo. Os reflexos são de igual magnitude sobre as avaliações com e sem flexibilidade. A total aversão à incerteza no sentido Knight agravou as exigências sobre o projeto em cada estágio, na medida em que deslocou os valores de mercado esperados em cada desempenho técnico para os valores mínimos das distribuições futuras.
Como a aversão à incerteza, por premissa, não afeta os custos do projeto, a quantidade de células com opção ótima Parar aumentou, contaminando o nó inicial (comparar as Figuras 4a e 5a).

0 quadro da Tabela 4 detalha os cálculos nos termos do quadro de relação entre as variáveis apresentado na Tabela 3, justificando as decisões

(a)

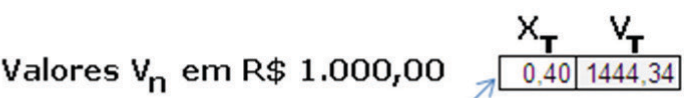

Árvore com flexibilidade (VPL Flex)

VPL Flex $=R \$ 162,25$

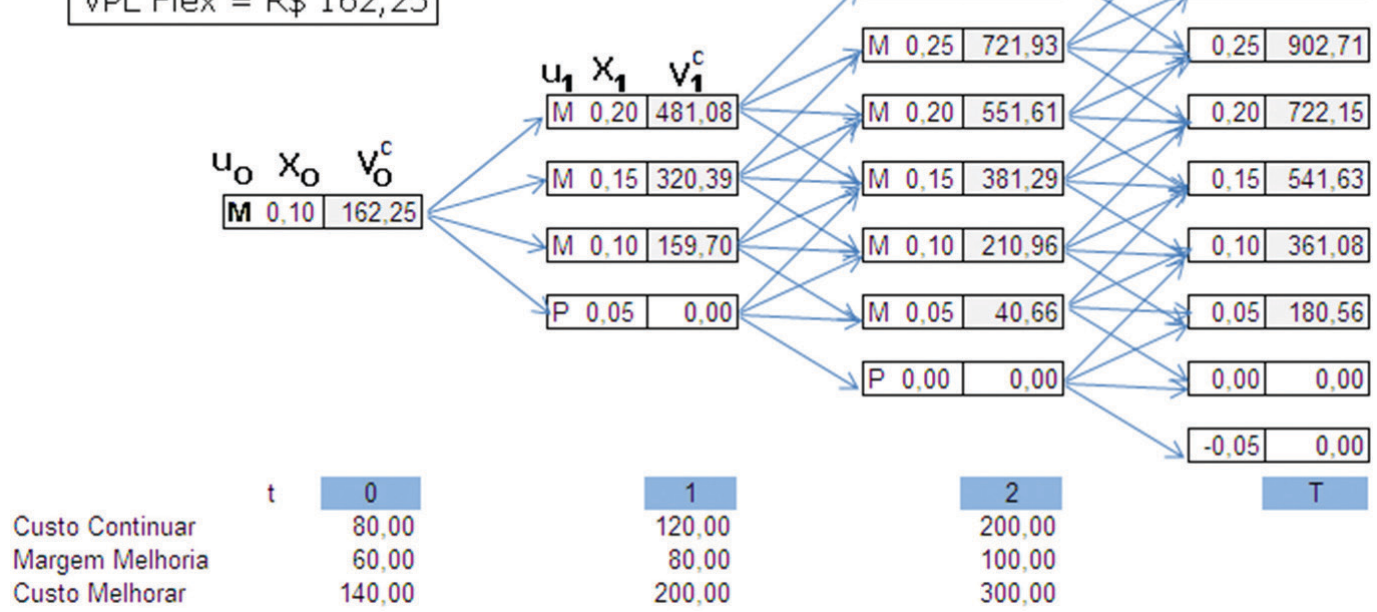

(b)

Valores $V_{n}$ em $R \$ 1.000,00$\begin{tabular}{cc|c|}
$X_{\mathbf{T}}$ & $V_{\mathbf{T}}$ \\
\cline { 2 - 3 } & 0,40 & 1444,34 \\
\hline
\end{tabular}

Árvore sem flexibilidade (VPL)

$\mathrm{VPL}=\mathrm{R} \$-68,03$

\begin{tabular}{l|l|}
0,35 & 1263,79 \\
\hline
\end{tabular}

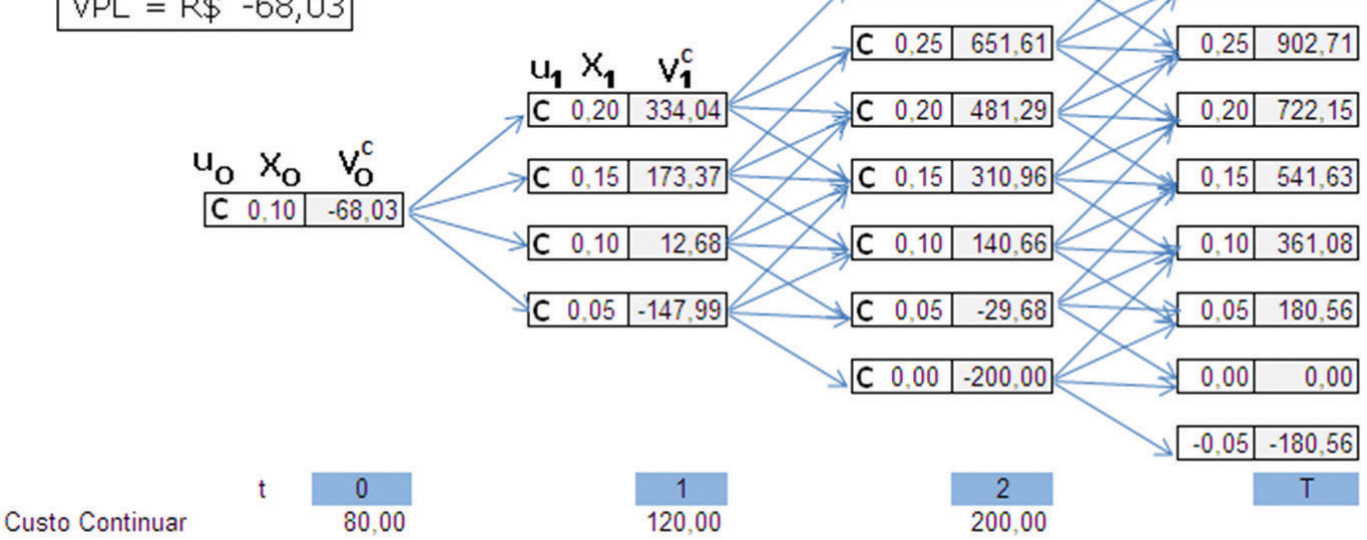

Figura 4. a. Avaliação de projeto de P\&D por Modelo de Opções Reais ( $c=0)$, com flexibilidade. b. Avaliação de projeto de P\&D por Modelo de Opções Reais (c = 0), sem flexibilidade. 
ótimas apresentadas nos nós iniciais das árvores com flexibilidade, tanto para $\mathrm{c}=0$ como para $\mathrm{c}=1$. As células marcadas com um grande $X$ apresentam valores que não cumpriram a relação que determina uma escolha ótima, as demais mostram o porque do cumprimento da relação. Pode-se observar que quando c $=1$ são cumpridas as relações para as opções Parar e Continuar, mas a primeira é preferencial, tal como foi explicado na seção 3.1.

Os diferentes resultados ótimos para quando $\mathrm{c}=0$ e $\mathrm{c}=1$ mostram que um mesmo projeto pode ser avaliado de forma diferente por decisores com percepções diferentes (e extremamente opostas), quando se leva em conta a aversão à incerteza no

(a)

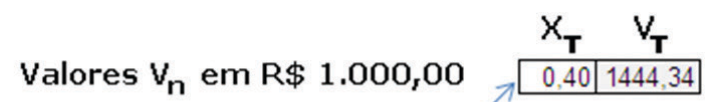

Árvore com flexibilidade (VPL Flex)

VPL Flex $=R \quad 0,00$
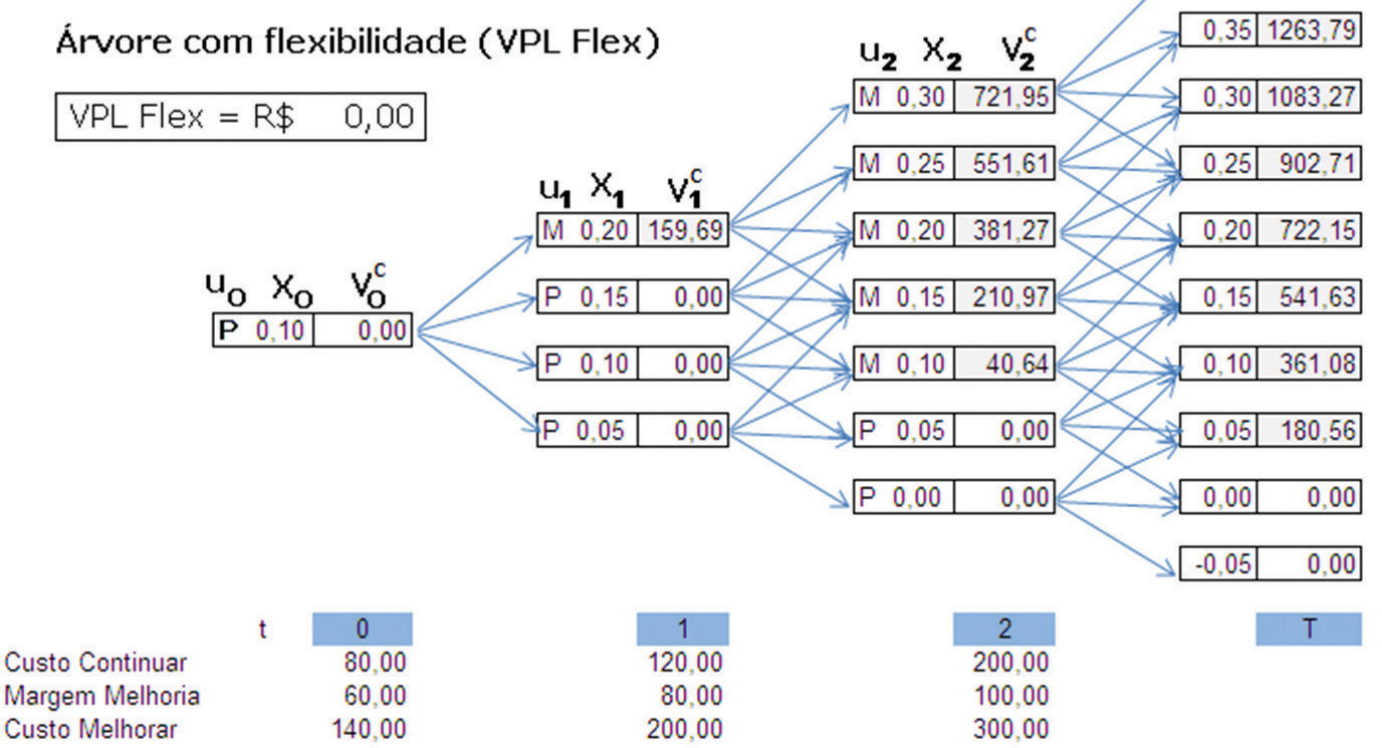

(b)

Valores $V_{n}$ em R \$ 1.000,00 \begin{tabular}{ccc}
$X_{\mathbf{T}}$ & $V_{\mathbf{T}}$ \\
\cline { 2 - 3 } & 0,40 & 1444,34 \\
\hline
\end{tabular}

Árvore sem flexibilidade (VPL)

$\mathrm{VPL}=\mathrm{R} \$-522,81$
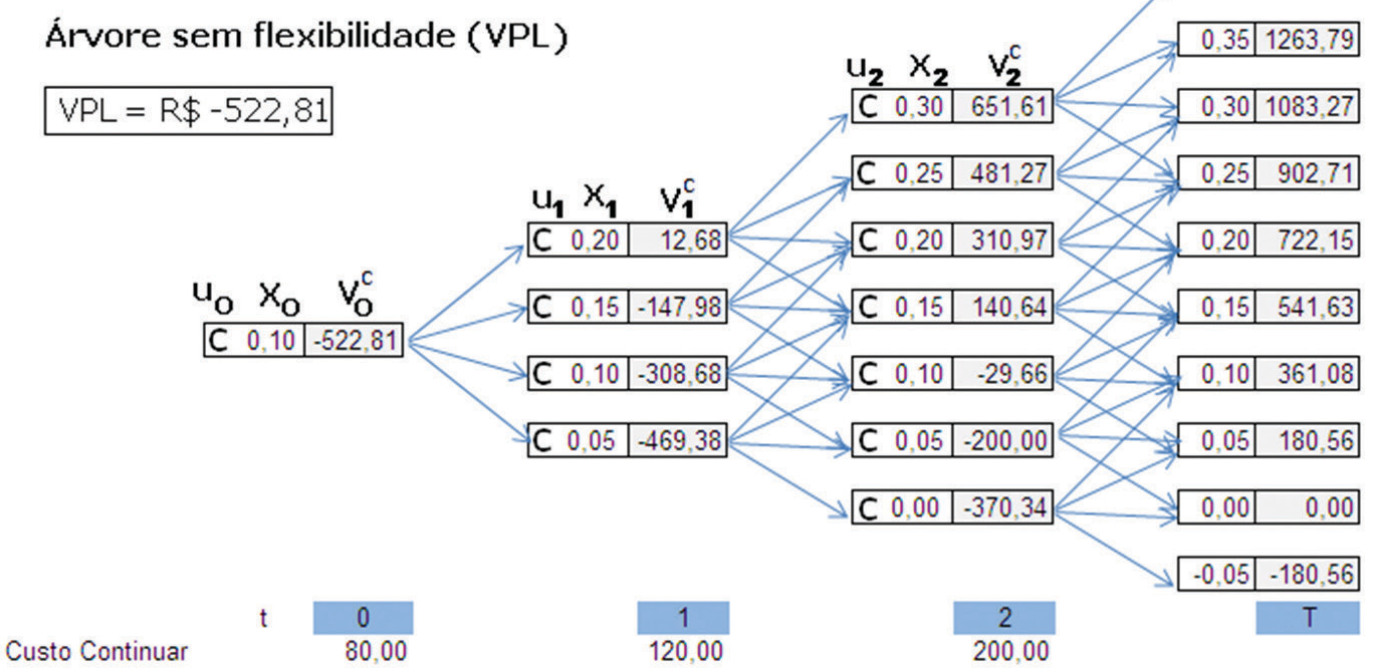

Figura 5 a. Avaliação de projeto de P\&D por Modelo de Opções Reais $(c=1)$, com flexibilidade. b. Avaliação de projeto de P\&D por Modelo de Opções Reais ( $c=1)$, sem flexibilidade. 
Tabela 4. Cálculos do projeto, com destaque para as células das decisões ótimas.

\begin{tabular}{|c|c|c|}
\hline $\begin{array}{c}u_{t} \\
\text { Decisão } \\
\text { otima }\end{array}$ & $\begin{array}{c}c=0 \\
\text { Sem aversão à } \\
\text { incerteza (puro RISCO) }\end{array}$ & $\begin{array}{c}\mathrm{c}=1 \\
\text { Com total aversão } \\
\text { à incerteza } \\
\text { (pura INCERTEZA) }\end{array}$ \\
\hline \multirow[t]{2}{*}{ PARAR } & $\begin{array}{l}\mathrm{R} \$ 302,25 \leq \mathrm{R} \$ 140,00 \\
\mathrm{R} \$ 151,13 \leq \mathrm{R} \$ 80,00\end{array}$ & $\begin{array}{c}\mathrm{R} \$ 0,00 \leq \mathrm{R} \$ 140,00 \\
\mathrm{e} \\
\mathrm{R} \$ 0,00 \leq \mathrm{R} \$ 80,00 \\
\mathrm{~V}_{\mathrm{t}}{ }^{\mathrm{C}} \mathrm{M}<0 \text { e } \mathrm{V}_{\mathrm{t}}{ }^{\mathrm{C}} \mathrm{C}<0\end{array}$ \\
\hline & \multicolumn{2}{|c|}{$\operatorname{Max}\left(V_{0}^{C} M, V_{0}^{C} C, 0\right)=0$} \\
\hline \multirow[t]{2}{*}{ MELHORAR } & $\begin{array}{c}\mathrm{R} \$ 151,13 \\
> \\
\mathrm{R} \$ 60,00 \\
\mathrm{~V}_{\mathrm{t}}^{\mathrm{c} M}=\mathrm{R} \$ 162,25 \\
\end{array}$ & \\
\hline & \multicolumn{2}{|c|}{$\operatorname{Max}\left(V_{0}^{C} M, V_{0}^{C} C, 0\right)=V_{0}^{C} M$} \\
\hline \multirow[t]{2}{*}{ CONTINUAR } & $R$ & $\begin{array}{c}\mathrm{R} \$ 0,00 \\
\leq \\
\mathrm{R} \$ 60,00 \\
\text { Obs.: } \mathrm{Max}\left(\mathrm{V}_{\mathrm{t}}^{\mathrm{C}} \mathrm{M}, \mathrm{V}_{\mathrm{t}}^{\mathrm{C}} \mathrm{C},\right. \\
0) \neq \mathrm{V}_{\mathrm{t}}{ }^{\mathrm{C}} \mathrm{C} ! \\
\mathrm{V}_{\mathrm{t}}^{\mathrm{c} C} \mathrm{C}=\mathrm{R} \$-80,00\end{array}$ \\
\hline & \multicolumn{2}{|c|}{$\operatorname{Max}\left(V_{0}^{C} M, V_{0}^{C} C, 0\right)=V_{0}^{C} C$} \\
\hline
\end{tabular}

sentido de Knight. A base de cálculo para o tratamento do risco é a mesma, o que difere é somente a postura diante da incerteza.

Acontece radical mudança de decisão quando a máxima aversão à incerteza é introduzida na avaliação, surge então naturalmente a pergunta: Sob que grau de aversão à incerteza, partindo de $\mathrm{c}=0$, a decisão ótima se modificaria? Interessante então examinar um exercício de sensibilidade sobre o grau de aversão à incerteza do agente decisor.

No caso desse projeto, por meio do recurso "atingir meta" do Excel, chega-se ao valor de c $=0,36$ para a decisão ótima mudar de Melhorar para Parar. Não houve trânsito ótimo pela opção Continuar, reflexo da configuração particular de custos e valores esperados futuros para essa opção, no caso específico desse projeto.

Os resultados da sensibilidade podem ser visualizados na Figura 6. Verifica-se que o valor da opção é sempre positivo e não decrescente. Os valores de VPL são deprimidos fortemente na medida em que aumenta o grau de aversão à incerteza do decisor. Percebe-se, provavelmente devido aos ganhos modestos proporcionados por uma inovação incremental em mercado com margens comprimidas, que a tolerância à incerteza implícita é pequena e que o projeto só é viável na presença da opção Melhorar.

Interpretando esse resultado, o "ambiente mental" decisório que envolve a avaliação desse projeto é algo sensível à ocorrência de eventos inesperados, que fujam à aditividade em que se baseia o seu tratamento de risco. 0 resultado dessa sensibilidade sobre o parâmetro c deriva intrinsecamente de toda a parametrização estabelecida para o projeto, envolvendo: a evolução de sua variável técnica $\left(X_{t}\right)$, que determina a construção da árvore de incerteza, de seus valores finais de mercado (curva de payoff, ou $\mathrm{V}_{\mathrm{T}}$ ), dos valores dos nós intermediários $\mathrm{V}_{\mathrm{t}}^{\mathrm{c}}$ calculados por backward induction, dos custos das opções, da taxa de juros livre de risco, das probabilidades aditivas de sucesso e insucesso, da quantidade de estágios, da duração do projeto etc. Obviamente, valores diferentes para esses parâmetros poderão determinar uma tolerância implícita maior a situações adversas não desejadas, considerando a premissa de que o agente econômico é avesso à incerteza e prima por ações mais prudentes quando se depara com ela.

Fosse diversa essa configuração de parâmetros, teríamos certamente outro comportamento, como por exemplo, no caso apresentado na Figura 7. Esse projeto fictício sugere uma tolerância maior à incerteza, com trânsito ótimo pela opção Continuar antes de se chegar ao ótimo na opção Abandonar (Parar). Certamente não é fácil estimar a aversão à incerteza de um decisor antes que ele tome propriamente uma decisão sobre um determinado projeto. Essa aversão, que é específica de cada projeto, pode variar de acordo com a ambiência, ou pode ser constante ao longo de um certo tempo, refletindo princípios individuais ou organizacionais. Esse aspecto é algo a ser ainda investigado.

Uma vez tomada uma decisão, podemos avaliar uma espécie de aversão à incerteza "revelada" do decisor para o projeto, na medida em que sua decisão se desvia de um ótimo calculado pelo modelo de tratamento de risco.

Por exemplo: seja o projeto fictício apresentado na Figura 7. Toda a parametrização de sua avaliação em risco técnico e de mercado reflete uma postura de neutralização desses riscos combinados. Se esse decisor seguir à risca o ótimo determinado pela avaliação, que é a opção Melhorar (iniciar com melhora), podemos afirmar, seguramente, que a aversão à incerteza revelada por esse decisor não será maior que 0,45 para esse projeto (valor de c em que a decisão passa de Melhorar para Continuar).

No entanto, assim como pode ser 0 , também pode ser 0,3 ou outro valor, até 0,45 . Agora, caso ele não siga o ótimo recomendado pelo modelo e, em vez de Mellhorar opte por apenas Continuar, sua aversão à incerteza revelada estará entre $0,45 \mathrm{e}$ 0,65 , garantindo que seja maior que 0 . Um desvio de decisão desse tipo sugere uma escolha não racional, ou subótima, do ponto de vista do tratamento de risco (sem considerar a Incerteza Knightiana). Essa 


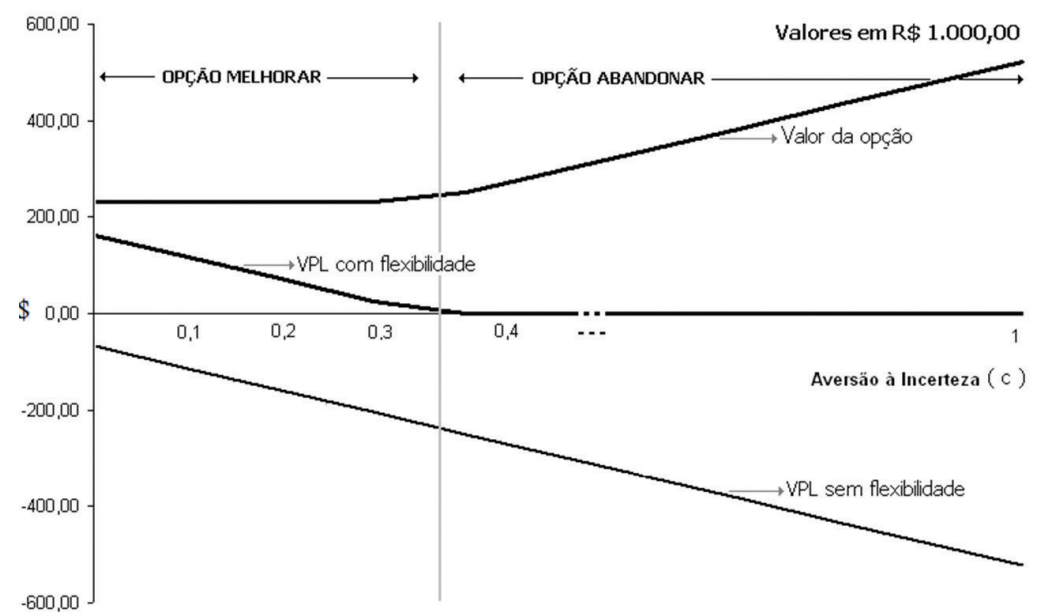

Figura 6. Sensibilidade sobre os VPLs a variações na aversão à incerteza.

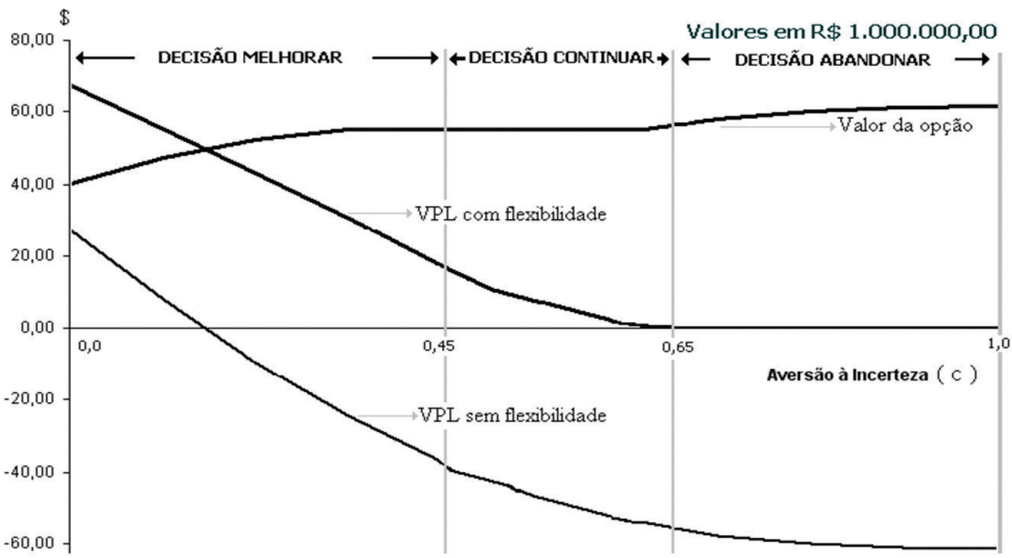

Figura 7. Sensibilidade sobre os VPLs a variações na aversão à incerteza - exemplo.

indicação é forte e sugere que o "verdadeiro" valor do projeto não é o seu VPL FLEX calculado $\operatorname{com} \mathrm{c}=0$, mas um VPL FLEX menor, com c entre 0,45 e 0,65.

Chegaremos a um intervalo de valor de VPL FLEX que seja aderente ao resultado final do processo decisório, respeitando a regra de decisão que determina a ação de seguir com o projeto (Melhorando ou Continuando) quando o VPL FLEX for maior que 0 . Finalmente, caso ele opte por Abandonar, sua aversão à incerteza revelada para o projeto será de, no mínimo, 0,65, e o VPL FLEX do projeto igual a 0 , sendo esse valor perfeitamente aderente à decisão tomada, pela regra de decisão do modelo.

Evidentemente, o exemplo da Figura 7 representa um projeto um tanto mais robusto (escala em $\mathrm{R} \$$ milhão) e mais típico de projetos de $\mathrm{P} \& \mathrm{D}$ envolvendo lançamento de produtos inovadores no mercado. Essa configuração fictícia de um projeto demonstra uma certa tolerância à Incerteza Knightiana, inclusive para o seu VPL sem flexibilidade, que apresenta valores positivos para valores baixos do parâmetro c.

\section{Conclusões e sugestões para novas pesquisas}

Com a introdução formal do tratamento da aversão à incerteza no sentido de Knight, o Modelo de Opções Reais voltado para P\&D ganha um novo e poderoso elemento, que possibilita uma consideração de teor mais tácito na valoração, ligado fortemente ao momento da tomada de decisão do agente sobre um projeto. Esse novo elemento contribui para um melhor entendimento das decisões tomadas quando essas não seguem o ótimo recomendado pelo tratamento convencional de risco. Com base em experiência própria, afirmamos que não são escassos os casos de projetos de P\&D, sobretudo 
de natureza incremental, que são abandonados ou postergados por grandes centros de pesquisa industriais de setores tecnologicamente maduros (que são pouco intensivos em $P \& D$ ), a despeito de rigorosas e favoráveis avaliações de risco, com o emprego de avançadas técnicas. Uma vez não seguida a opção ótima sugerida por uma visão de puro risco, há indícios de existência de aversão à Incerteza Knightiana nesse agente, e o valor final do projeto, se aderente à regra básica de decisão do VPL, seria diferente.

Talvez seja impróprio considerar o VPL FLEX pós-decisão (VPL FLEX de Choquet) como um valor que venha a representar a viabilidade econômica do projeto, uma vez que entra no cálculo um elemento externo à sua valoração intrínseca, que diz respeito à postura final do decisor diante de um quadro de incerteza. Talvez seja mais apropriado chamá-lo de "VPL da decisão sob Incerteza Knightiana", ou outra denominação mais representativa de sua real significação, e não considera-lo de forma única. Minimamente, essa abordagem possibilita um melhor entendimento da decisão sob um ponto de vista de sua valoração.

A aversão à incerteza no sentido de Knight não é de fácil quantificação. 0 exercício de valoração realizado, juntamente com um resultado final de decisão sobre um projeto, dá indícios de seu valor em intervalos. Já se ela reflete uma postura individual, departamental, corporativa etc., se é constante ou variável no curto prazo e que condições do ambiente a influenciam são questões relevantes a serem investigadas em pesquisas futuras. 0 parâmetro c, que varia de 0 a 1, não possui ainda uma escala qualitativa rigorosamente estabelecida, que descreva detalhadamente sua significação. Ser 0,6 avesso à incerteza é muito diferente de ser apenas 0,4 ? A depender da avaliação do projeto em risco, a indicação de decisão pode mudar de um valor para outro, mas que significariam propriamente um 0,6 e um 0,4? A título de sugestão de partida, imaginamos uma escala intervalar qualitativa para uma empresa industrial, em setor pouco intensivo em $\mathrm{P} \& \mathrm{D}$, na seguinte forma:

Observando a Figura 8 podemos afirmar, seguramente, que $\mathrm{c}=0$ significa indiferença total à Incerteza Knightiana e $\mathrm{c}=1$, aversão máxima a ela. Com intervalos a cada 0,125 unidades, foram delimitados blocos a título de sugestão de categorização.

No exemplo do projeto de leite et al. (2012) aqui verificado, caso o projeto seja de fato abandonado, o agente decisor terá sido pelo menos 0,36 avesso à Incerteza Knightiana em sua avaliação, caindo minimamente na categoria Moderada. A significação dessas categorias e as possíveis inferências dela resultantes são também um tema interessante para

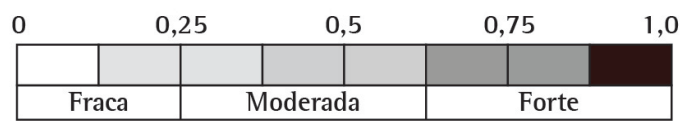

Figura 8. Escala sugerida para o parâmetro c - aversão à Incerteza Knightiana.

pesquisas futuras. Contudo, não temos ainda como calcular o c pontualmente. Nesse projeto, o agente que toma a decisão de cancelá-lo poderia ser também representado por um $\mathrm{c}=1$, pois apenas podemos concluir que o c do agente é pelo menos 0,36 !

Sob que condições poderia esse parâmetro ser considerado ex ante na avaliação de projetos sob incerteza, orientando as decisões de uma forma mais abrangente? Uma nova e devastadora crise econômica global justificaria sua adoção? Serviria apenas para identificar o quão afastadas de uma "aditividade" desejável no espaço probabilístico as mensurações de risco estão para um agente em situações particulares? Em vez de aversão à incerteza, poderíamos considerar situações em que as posturas fossem favoráveis, ou "amantes" da incerteza no sentido de Knight, com um viés no melhor resultado da distribuição futura? Esse parece ser um comportamento típico de muitas empresas que investem largamente em pesquisa, em setores mais intensivos em P\&D. São outras interessantes sugestões que deixamos para desenvolvimentos em pesquisas futuras.

\section{Referências Bibliográficas}

Brealey, R., \& Myers, S. (2003). Principles of Corporate Finance (7th ed.). Nova York: Mc Graw-Hill.

Choquet, G. (1953). Theory of Capacities. Annales de l'institut Fourier, 5, 131-295. http://dx.doi.org/10.5802/aif.53

Coimbra-Lisboa, P. C. (2008). Uma Introdução à Escolha Individual Sob Incerteza Knightiana, notas de aula do curso sobre Teoria dos Jogos, primeira parte. Rio de Janeiro: EPGE/FGV.

Dellacherie, C. (1972). Capacités et Processus Stochastiques. Berlin: Springer-Verlag.

Dempster, A. (1967). Upper and Lower Probabilities Induced by a Multivalued Mapping. Annals Mathematical Statistics, 38, 205-247. http://dx.doi.org/10.1214/ aoms/1177698950

Dixit, A. K., \& Pindyck, R. S. (1994). Investment Under Uncertainty. Princeton: Princeton University Press. PMCid:PMC1887235.

Dow, J., \& Werlang, S. R. C. (1992). Uncertainty Aversion, Risk Aversion, and the Optimal Choice of Portfolio. Econometrica, 60, 197-204. http://dx.doi. $\operatorname{org} / 10.2307 / 2951685$

Ellsberg, D. (1961). Risk, Ambiguity and the Savage Axioms. Quarterly Journal of Economics, 75, 643-669. http:// dx.doi.org/10.2307/1884324 
Gilboa, 1., \& Schmeidler, D. (1989). Maxmin Expected Utility with Non-unique Prior. Journal of Mathematical Economics, 18, 141-153. http://dx.doi. org/10.1016/0304-4068(89)90018-9

Gilboa, 1. (2009). Theory of Decision Under Uncertainty. New York: Cambridge University Press. http://dx.doi. org/10.1017/CB09780511840203

Huchzermeier, A., \& Loch, C. H. (2001). Project management under risk: Using real options approach to evaluate flexibility in R\&D. Management Science, 47, 85-101. http://dx.doi.org/10.1287/mnsc.47.1.85.10661

Knight, F. (1921). Risk, Uncertainty and Profit. Boston: Hougohton Mifflin. PMid:17864564 PMCid:PMC1399809

Leite, L. A. M., Teixeira, J. P., \& Samanez, C. P. (2012). Ex ante Economic Assesment in Incremental R\&D Projects: Technical and Development Time Uncertainties Addressed By Real Options Theory. Pesquisa Operacional, 32(3), 617-642. http://dx.doi.org/10.1590/ S0101-74382012005000025

Neumann, V., \& Morgenstern, J. (1947). Theory of Games and Economic Behaviour. Princeton: Princeton University Press.

Petrobras, \& Universidade Federal de Minas Gerais. (2008). Desenvolvimento de Metodologia para a Avaliação de Projetos de P\&D Baseada na Teoria das Opções Reais - Relatório Final. Relatório técnico de projeto de P\&D. Rio de Janeiro: Petrobras.

Samanez, C. P. (2007). Gestão de Investimentos e Geração de Valor. Pearson Prentince Hall. São Paulo.

Santiago, L. P., \& Bifano, T. G. (2005). Management of R\&D projects under uncertainty: A multidimensional approach to managerial flexibility. IEEE Transactions on Engineering Management, 52, 269-280. http://dx.doi. org/10.1109/TEM.2005.844465
Santiago, L. P., \& Vakili, P. (2005). On the Value of Flexibility in R\&D Projects. Management Science, 51, 1206-1218. http://dx.doi.org/10.1287/mnsc. 1050.0387

Savage, L. J. (1954). The Foundations of Statistics (2nd ed.). New York: John Willey; Dover.

Shapley, L. S. (1972). Cores of convex Games. International Journal of Game Theory, 1, 11-26. http://dx.doi. org/10.1007/BF01753431

Schmeidler, D. (1982). Subjective Probability and Expected Utility without Additivity (pp. 84-112). CARESS, Working Paper.

Schmeidler, D. (1989). Subjective Probability and Expected Utility without Additivity. Econometrica, 57, 571-587. http://dx.doi.org/10.2307/1911053

Shafer, G. (1976). A Mathematical Theory of Evidence. Princeton: Princeton University Press. PMCid:PMC2490625.

Silva, T. A. 0., \& Santiago, L. P. (2009). New product development projects evaluation under time uncertainty. Pesquisa Operacional, 29, 517-532. http://dx.doi. org/10.1590/S0101-74382009000300003

Simonsen, M. H., \& Werlang, S. R. C. (1991). Subadditive probabilites and portfolio Inertia. Revista de Econometria, 11(1), 1-19.

Trigeorgis, L. (1996). Real Options, Managerial Flexibility and Strategy in Resource Allocation (2nd print.). London: MIT Press.

\section{Agradecimentos}

Esta pesquisa foi parcialmente apoiada pela Petrobras, CNPq, Capes, Fapemig e PUC-Rio.

\title{
Real Options under Knightian Uncertainty for Economic Evaluation of Research and Development (R\&D) Projects
}

\begin{abstract}
This article presents a quantitative treatment of uncertainty, in the classical sense of Frank Knight (1921), in an economic assessment model that is based on Real Options Theory. The Knightian concept draws a distinction between uncertainty and risk. Optimal decisions in real options models, once discarded by the decision makers, become suboptimal choices, suggesting the presence of Knightian Uncertainty elements in the decision environment. Suboptimal decisions contradict the basic NPV rule, as the decision makers are rational by assumption. This results in a so-called "ex-post NPV" that harmonizes with that choice, according to the decision rule, by quantitatively determining a degree of uncertainty aversion. By adopting the Choquet Expected Value and a parameter representing uncertainty aversion, and by using the concept of non-additive probabilities, we obtain approximations of "postdecision NPVs" and determine the degrees of uncertainty aversion. The proposed formulation is used to evaluate an R\&D project.
\end{abstract}

\section{Keywords}

Decision under uncertainty. Real Options in R\&D. Economic evaluation of R\&D projects. R\&D incremental. Knightian Uncertainty. 